\title{
Probabilistic Graphical Model using Bayesian Networks for Predicting Clinical Outcome after Posterior Decompression in Patients with Cervical Spondylotic Myelopathy
}

\section{Sung Bae Park ( $\sim$ ddolbae01@naver.com )}

Seoul National University Seoul Metropolitan Government Boramae Medical Center https://orcid.org/0000-0002-4652-3056

\section{Sohee Oh}

Seoul National University Seoul Metropolitan Government Boramae Medical Center

\section{Changwon Yoo}

Robert Stempel College of Public Health and Social Work

\section{Dong Ah Shin}

Yonsei University College of Medicine

\section{Sun-Ho Lee}

Sungkyunkwan University School of Medicine

Hee-Jin Yang

Seoul National University Seoul Metropolitan Government Boramae Medical Center

\section{Technical advance}

Keywords: Bayesian network, cervical myelopathy, graphical model, prediction, probability

Posted Date: July 29th, 2020

DOI: https://doi.org/10.21203/rs.3.rs-45605/v1

License: (9) This work is licensed under a Creative Commons Attribution 4.0 International License. Read Full License 


\section{Abstract}

\section{Background}

The objective of this study was to develop a probabilistic graphical model (PGM) to show the personalized prediction of clinical outcome in patients with cervical spondylotic myelopathy (CSM) with different clinical conditions after posterior decompression and to use the PGM to identify causal predictors of the outcome.

\section{Methods}

We included data from 59 patients who had undergone cervical posterior decompression for CSM. The candidate predictive parameters were age, sex, body mass index, trauma history, symptom duration, preoperative and last Japanese Orthopaedic Association (JOA) scores, gait impairment, claudication, bladder dysfunction, Nurick grade, American Spinal Injury Association (ASIA) grade, smoking, diabetes mellitus, cardiopulmonary disorders, hypertension, stroke, Parkinson disease, dementia, psychiatric disorders, arthritis, ossification of the posterior longitudinal ligament, cord signal change in T1-weighted images, postoperative kyphosis, and cord compression ratio. Statistical and Bayesian network analyses were used to create the PGM and identify predictive factors.

\section{Results}

In multiple linear regression analysis, preoperative JOA score, presence of a psychiatric disorder, and ASIA grade were identified as significant factors associated with the last JOA score. Dementia, sex, preoperative JOA score, and gait impairment were causal factors in the PGM with 93.2\% accuracy. Sex, dementia, and preoperative JOA score were direct causal factors related to the last JOA score. Being female, having dementia, and a low preoperative JOA score were significantly related to having a low last JOA score. The PGM showed that preoperative JOA score and sex did not affect the last JOA score in patients with dementia. The probability of having a high last JOA score was higher in men with a high preoperative JOA score than in women with the same preoperative state ( $74 \%$ vs. $2 \%$, respectively).

\section{Conclusions}

The causal predictors of surgical outcome for CSM were sex, dementia, and preoperative JOA score. Use of the PGM with the Bayesian network may be useful personalized medicine tool for predicting the outcome for each patient with CSM.

\section{Background}

Cervical spondylotic myelopathy (CSM) is a common degenerative spine disorder that can cause spinal cord dysfunction [1]. It is controversial whether decompressive surgery should be performed in patients with CSM $[2,3]$. The natural history of CSM shows that the symptoms of myelopathy will worsen over Loading [MathJax]/jax/output/CommonHTML/jax.js ff patients [4, 5]. Some studies have reported that this surgery 
can arrest the natural history of CSM and disease progression, and prevent further neurological decline [6, 7]. Therefore, surgical decompression to remove the compressive pathology and increase the space available for the spinal cord is considered to be an acceptable treatment for CSM. However, surgical decompression surgery does not always improve the clinical outcome with patients with CSM.

To address the limitations of surgical decompression, researchers have studied the key factors that predict the surgical outcome for patients with CSM. The independent factors identified as predictors including age, Japanese Orthopaedic Association (JOA) scores, Nurick grade, impaired gait, duration of symptom, diabetes, psychiatric comorbidities, sex, smoking, changes in T1-weighted MRI (T1MRI) images, and the compression ratio of the spinal cord [1, 8-12]. It is unclear which patients will experience meaningful improvement after surgical decompression, and it is a challenge for spine surgeons to predict who is likely to benefit the most from surgical decompression.

A prediction tool would be valuable to creating an expectation for the surgical outcome for individual patients with CSM and for supporting the surgeon's decision. To identify statistically meaningful predictive factors from previous studies, multivariate logistic regression analyses have been conducted to strengthen the results of univariate analyses [13]. However, previous statistical analyses have several disadvantages. Although predictive factors may correlate significantly with other factors, the logistic regression analyses shows only parallel relationships between factors and cannot identify causal interactions [14]. In addition, each outcome of interest should be trained using its own individual model [15]. It is difficult to obtain sufficient data for thorough statistical analysis and the treatment guidelines for some kinds of diseases are not universally accepted or may change rapidly (e.g., malignant diseases or rare diseases) [15]. Surgeons sometimes ask for expert opinion in difficult cases.

It would be helpful to have a prediction tool to overcome obstacles such as incomplete, insufficient, or missing data and to identify causal relationships between factors. Such a prediction tool may help in the treatment of CSM by improving the probability of a successful treatment outcome. Given the limitations of the regression-based approach, it may not be suitable for risk prediction for individual patients using what-if scenarios and effect-to cause reasoning in the era of precision and personalized medicine [16].

Using machine learning algorithms, Bayesian networks (BNs) can identify casual relationships between variables and show predictive inferences using probabilistic graphical modeling (PGM) [17]. PGM using BNs can be used to predict risk at the individual patient level and to show multiple outcomes and exposures in a single model [15]. Several recent articles have described the use of BNs in medical prediction models $[15,18]$. In this study, we applied BNs to facilitate understanding of the interaction between surgical outcome for CSM and clinical factors associated with the clinical outcome after posterior decompressive surgery. We hoped to show that a PGM using BNs provides a way to predict the clinical outcome of CSM after posterior decompression and to help the spine surgeon make decisions about the surgical treatment of patients with CSM.

\section{Methods}

Loading [MathJax]/jax/output/CommonHTML/jax.js 
After receiving approval from our Clinical Research Ethics Board (number: 20200330/30-2020-20/043), the medical records of the 59 consecutive patients who underwent posterior decompressive surgery at our hospital between 2012 and 2016 were reviewed retrospectively. The patients were diagnosed with CSM on the basis of their clinical signs and symptoms of cervical myelopathy and concordant MRI findings of cervical cord compression with or without signal changes because of spondylosis. The 59 patients underwent posterior decompressive surgery with a minimum follow-up of 12 months. No patients had demyelinating disease, tumors, previous cervical surgery, or intradural pathology.

Twenty-five variables were assessed: last follow-up JOA score (LastJOA), sex, age, body mass index (BMI), cervical trauma history, smoking, diabetes mellitus (DM), hypertension, arthritis, psychiatric disorder, stroke, Parkinson's disease, dementia, cardiopulmonary disease, ossification of the posterior longitudinal ligament (OPLL), claudication, gait disturbance, bladder dysfunction, symptom duration before surgery, preoperative JOA score (PreJOA), American Spinal Injury Association (ASIA) grade, Nurick grade, cervical cord signal changes in T1MRI images, postoperative kyphosis (PostKyphosis) identified in a postoperative X-ray, and cord compression ratio observed on the preoperative cervical MRI (CR). The CR was calculated by dividing the sagittal diameter by the transverse diameter of the spinal cord at the most compressed level. A smaller CR value indicated greater cervical compression. Twenty-three of the 25 variables, excluding LastJOA and PostKyphosis, were evaluated preoperatively. Thirteen of these variables are known as clinical risk factors associated with surgical outcome in patients with CSM: T1MRI, claudication, arthritis, cardiopulmonary disease, psychiatric disorder, symptom duration, gait impairment, bladder function, PostKyphosis, smoking, age, PreJOA, and CR $[1,10,11,19]$.

We used statistical analysis to search for factors significantly associated with the LastJOA and compared the factors identified in the PGM using BN analysis of the 25 variables for the 59 patients enrolled in the present study.

\section{Statistical analysis}

To identify factors associated with the LastJOA, we selected meaningful variables using least absolute shrinkage and selection operator (LASSO) analysis. We then used multivariable logistic regression with backward elimination for the variables with $>80 \%$ selection rates in the LASSO analysis. All statistical analyses were performed using IBM SPSS Statistics (version 26; IBM Corp., Armonk, NY, USA).

\section{Methods}

We included data from 59 patients who had undergone cervical posterior decompression for CSM. The candidate predictive parameters were age, sex, body mass index, trauma history, symptom duration, preoperative and last Japanese Orthopaedic Association (JOA) scores, gait impairment, claudication, bladder dysfunction, Nurick grade, American Spinal Injury Association (ASIA) grade, smoking, diabetes mellitus, cardiopulmonary disorders, hypertension, stroke, Parkinson disease, dementia, psychiatric disorders, arthritis, ossification of the posterior longitudinal ligament, cord signal change in T1-weighted 
images, postoperative kyphosis, and cord compression ratio. Statistical and Bayesian network analyses were used to create the PGM and identify predictive factors.

\section{Results}

The average age and follow-up duration of the patients whose data were included were $65 \pm 1.7$ years and $33 \pm 13.8$ months, respectively, and $30.5 \%(n=18)$ were women. The mean preoperative and postoperative JOA scores were $9 \pm 3.4$ and $12 \pm 3.2$, respectively. The surgical treatment seemed to be effective in these patients with CSM. The values for the PreJOA were discretized as follows: $<6(z<-1)$, 6-12 $(-1 \leq z \leq 1)$, and $>12$ ( $z>1$ ) were expressed as 0 (low score), 1 (normal range), and 2 (high score), respectively. The LastJOA was expressed as 0 (JOA score $<9), 1$ ( $9 \leq$ JOA score $\leq 15)$, and 2 (JOA score $>15)$.

Before surgery, 36 patients $(68 \%)$ had gait disturbance and 17 patients $(28 \%)$ had bladder symptoms. ASIA grades D and E were observed in 30 and nine patients, respectively. Hypertension and OPLL were found in $54 \%$ and $52 \%$ of all patients, respectively. Fewer than one-third of the 59 patients had a comorbidity such as DM, psychiatric disease, stroke, arthritis, cardiopulmonary disease, Parkinson disease, or dementia (Table 1). 
Table 1

Patients Characteristics

\begin{tabular}{|llll|}
\hline Characteristics & $\begin{array}{l}\text { Total (N= } \\
\text { 59) }\end{array}$ & & Total (N = 59) \\
\hline Mean age, years & $65 \pm 11.7$ & ASIA grade (A/B/C/D/E) & $\begin{array}{l}1 / 1 / 18 / \\
30 / 9\end{array}$ \\
\hline Female sex & $18(30.5 \%)$ & Nurick grade (1/2/3/4/5) & $11 / 20 / 9 / 15 / 4$ \\
\hline Mean follow-up duration, months & $33 \pm 13.8$ & Trauma Hx. (yes) & $18(30.5 \%)$ \\
\hline Symptom duration, month & $7 \pm 11.0$ & Smoking (yes) & $14(23.7 \%)$ \\
\hline Preoperative JOA score & $9 \pm 3.4$ & Diabetes mellitus (yes) & $19(32.2 \%)$ \\
\hline Last JOA score & $12 \pm 3.2$ & Psychiatric disease (yes) & $3(5.1 \%)$ \\
\hline $\begin{array}{l}\text { Cord signal change in T1 image of } \\
\text { MRI (yes) }\end{array}$ & $5(8.5 \%)$ & Stroke (yes) & $7(11.9 \%)$ \\
\hline Cord compression ratio & $0.3 \pm 0.11$ & Arthritis & $8(13.6 \%)$ \\
\hline $\begin{array}{l}\text { Postoperative kyphosis (yes) } \\
\left.\text { Body mass index (kg/m }{ }^{2}\right)\end{array}$ & $16(27.1 \%)$ & $\begin{array}{l}\text { Cardiopulmonary disease } \\
\text { (yes) }\end{array}$ & $10(16.9 \%)$ \\
\hline $\begin{array}{l}\text { Gait disturbance (yes) } \\
\text { Claudication (yes) }\end{array}$ & $23 \pm 5.6$ & Parkinson disease (yes) & $1(1.7 \%)$ \\
\hline $\begin{array}{l}\text { Bladder symptom(yes) } \\
\text { JOA, Japanese Orthopaedic Association; ASIA, American Spinal Injury Association; OPLL, ossification } \\
\text { of posterior longitudinal ligament }\end{array}$ & $36(61 \%)$ & Dementia (yes) & $4(6.8 \%)$ \\
\hline
\end{tabular}

The LASSO analysis identified selection rates $>80 \%$ for the PreJOA, ASIA grade, and psychiatric disorders as $97.8 \%, 88.0 \%$, and $83.0 \%$, respectively. Therefore, we included these three variables in the multiple linear regression, which showed that all were significantly related to the LastJOA $(p<0.0001,0.0020$, and 0.0291, respectively) (Table 2). 
Table 2

Multiple linear regression analysis of three factors

\begin{tabular}{|lllll|}
\hline Variable & Estimate & Std.error & P-value & adjR2 \\
\hline Preop JOA & 0.4963 & 0.0918 & $<0.0001$ & \multirow{2}{*}{0.6876} \\
\cline { 1 - 4 } AISA & & & 0.0020 & \\
\cline { 1 - 4 } B vs. A & 2.5037 & 2.6045 & 0.3409 & \\
\cline { 1 - 4 } C vs. A & 7.6602 & 2.0145 & 0.0004 & \\
\cline { 1 - 4 } D vs. A & 7.1412 & 2.1319 & 0.0015 & \\
\cline { 1 - 4 } E vs. A & 8.1078 & 2.3328 & 0.0010 & \\
\cline { 1 - 4 } psychiatric disorder & -2.6281 & 1.1711 & 0.0291 & \\
\hline JOA, Japanese Orthopaedic Association & & \\
\hline
\end{tabular}

\section{BN Analysis}

We report the three BN structures $\left(S^{1}, S^{2}\right.$, and $\left.S^{3}\right)$, $S^{\circ d}$, and the final structure $\left(S^{\prime}\right)$ chosen along with the results of the validation of $S^{f}$.

\section{BN with all variables}

Among the $12 \mathrm{BNs}$, the one with the best log-likelihood score showed significantly better data fit than the second-best BN (viz., $\frac{P\left(D^{1} \mid S^{1}\right)}{P\left(D^{1} \mid S^{1}\right)+P\left(D^{1} \mid S^{1}\right)}>99.999 \%$ where $S^{1}$ and $S^{\prime}$ are the first- and second-best $\mathrm{BNs}$, respectively, with 25 variables, and $D^{1}$ is the dataset with the same number of variables with 59 patients. In the follow-up BN learning comprising the $12 \mathrm{BNs}$, one $\mathrm{BN}\left(S^{1}\right)$ with 25 variables showed significantly better fit (>99.999\%) than the second-best BN. Sex, dementia, psychiatric disorders, and the PreJOA were the direct plausible cause (parents) of the LastJOA, and the ASIA grade was the plausible effect of the LastJOA. Gait impairment was the direct cause (coparent node) for the LastJOA and the ASIA grade (Fig. 2A). Subsequently, 16 variables within the second-degree MB of the LastJOA in the first $\mathrm{BN}\left(S^{1}\right)$ were selected in the second BANJO analysis. The second BN $\left(S^{2}\right)$ that best fit the datasets with 16 variables and 59 patients is shown in Fig. 2B. The parent variables of the LastJOA in the second BN $\left(S^{2}\right)$ were the same as those in the first BN $\left(S^{7}\right)$ except for psychiatric disease.

In the third BANJO analysis, five variables within the first-degree MB of the LastJOA in $S^{2}$ were selected. The 12 BNs in the third BANJO analysis had the same log-likelihood score (-222.6719) and structure. The final BN $\left(S^{3}\right)$ obtained after the third BANJO analysis is shown in Fig. 3A. In $S^{3}$, dementia, sex, and Loading [MathJax]/jax/output/CommonHTML/jax.js rents) of the LastJOA. Because the Order algorithm 
summarizes the significantly causal BN structures identified, the most likely summarized structure (Fig. 3B) shows a similar structure as that for $S^{3}$. The Order algorithm yielded the following order as the most probable: gait impairment, the PreJOA, sex, psychiatric disease, ASIA grade, and the LastJOA. However, the likelihood score of $S^{o d}$ was superior to that for $S^{3}$, which suggested that $S^{o d}$ reflected the relationship and causality between the LastJOA and other variables in the current data better than $S^{3}$. Therefore, $S^{o d}$ was selected as the final BN structure $\left(S^{\dagger}\right)$. In $S^{f}$ (Fig. 3B), sex, dementia, and PreJOA nodes were direct parents of the LastJOA node. Although gait impairment and ASIA grade correlated with the LastJOA, if the PreJOA was conditioned ("if we knew the PreJOA of the selected patient"), the information for gait impairment and AISA grade for the patient did not influence the LastJOA. Sex was a direct parent of both dementia and the LastJOA nodes, simultaneously.

\section{Learning causal $\mathrm{BN}$ parameters}

The parameters (probabilities) of the final $\mathrm{BN}\left(S^{\prime}\right)$ with six variables were learned from a new dataset that contained six variables and 59 patients (denoted as $D^{3}$ ) that had been extracted from the dataset $D^{1}$ containing the 25 variables from all of the 59 subjects (Fig. 4A). Changing the LastJOA conditioned as state 0 to state 2 increased the probabilities of state 2 in the PreJOA, not having dementia, and being male (Fig. 4B and C). Sex and the PreJOA were direct parent (causal) variables for the LastJOA. Conditioning the sex variable from state 0 (female) to state 1 (male) decreased the probability of state 0 and increased the probability of state 2 in the LastJOA (Fig. 5A and B). Men with a high PreJOA had a higher probability of a high LastJOA than did women with same preoperative state ( $74 \%$ vs. $2 \%$, respectively). This suggested that men with CSM may have a higher probability of a good outcome after posterior decompressive surgery than women with CSM.

Changing the PreJOA from state 0 to 2 increased the probability of state 2 in the LastJOA (Fig. 5C and D). Patients with a high PreJOA had a better outcome after the surgical treatment compared with those with a low PreJOA. Dementia seemed to predict a poor outcome of the LastJOA (Fig. 6A and B). With conditioned dementia as state 1 , a change in PreJOA did not influence the LastJOA (Fig. 6C and D). These findings suggest a 76\% probability of the LastJOA can be expected in men with CSM but without dementia and a PreJOA $>12$ (Fig. 7A.). By contrast, the probability of state 1 or 2 was estimated as $31 \%$ for the Last JOA (Fig. 7B).

3. Validation

We used LOOCV to evaluate the prediction accuracy of the final BN parameterized by $D^{3}$. This analysis produced a $72.6 \%$ value for correct predictions (257/354 for 6 . 59 cases). Using only direct causes (parents) of the LastJOA in the BN (i.e., $3 / 6$ variables), the prediction was 81.4\% (192/236 correct predictions for $4 \cdot 29$ cases). For only the LastJOA, prediction was 93.2\% (55/59 correct predictions for 1 . 59 cases). The AUC for predicting states $0,1,2$ for the LastJOA were $0.96,0.85$, and 0.79 in the final BN, respectively. 


\section{Discussion}

The final BN showed that, among the 24 factors used in the analysis, sex, dementia, the PreJOA, and gait impairment were causal factors associated with the LastJOA. ASIA grade was related to the LastJOA and was a child node of gait impairment. Although the final BN showed that dementia, sex, the PreJOA, AISA grade, and gait impairment were more closely related to the LastJOA than the other 19 factors, sex, dementia, and the PreJOA were direct causal factors for the LastJOA. In the multivariate analysis, the PreJOA, ASIA grade, and psychiatric disorder were significant predictors of the LastJOA. Having a psychiatric disorder was also a parent node of dementia in $S^{2}$. Including clinical information about comorbidity in patients with dementia showed that the comorbidity of having a psychiatric disorder did not influence the LastJOA (Fig. 2B). Therefore, having a psychiatric disorder was not included as a variable in the final $B N$ analysis. The final $B N$ structure reflected the statistical relationships between the LastJOA and three variables: the PreJOA, ASIA grade, and having a psychiatric disorder.

Psychiatric comorbidities, sex, PreJOA, ASIA grade, and gait impairment are significant factors related to surgical outcome [10-12]. Depression or bipolar disorder are significantly associated with clinical outcome assessed with the JOA score [11]. Although no study has reported statistical associations between dementia and surgical outcomes of patients with CSM, having dementia may influence cervical stenosis [23]. Because only four of 59 patients had dementia in the present study, dementia seemed not to be a significant variable in the statistical analysis. The first and second BN structures considered 25 and 16 variables simultaneously and showed that dementia was a direct parent node and having a psychiatric disorder was a parent node only of the LastJOA. In the present study, the final BN structure showed that the LastJOA was significantly related to the three direct causal factors sex, having dementia, and the PreJOA (Figs. 4-7). As a predictive model, the BN may provide a personalized prediction by predicting the probability of the target outcome according to the change in each factor for each patient.

Although the amount of clinical data is increasing and becoming more complicated, data to explain differences in phenotype are incomplete in the medical field, and there will always be uncertainty when analyzing data. It is difficult to use only logistic regression analysis to describe correlations because of the inability to identify causal relationships between predictors. Therefore, BN analysis, which is graphic and intuitive to the clinician, may help to identify layered and causal correlations between predictors more clearly than a graphical model $[13,20]$. The BN structure showing the entire network between variables may help to identify the organic relationships with a target variable and the important factors to focus on, and to determine the best ways to improve the clinical outcome. Furthermore, BNs with their associated methods are especially suited for reasoning with uncertainty [24]. Our model allows us to intuitively understand the causal correlations between the factors in the final $\mathrm{BN}\left(S^{\prime}\right)$ of the present study.

Although the present study showed an advantage of BN analysis over conventional analysis, our study has several limitations. First, we tried to identify 24 factors as closely related to the LastJOA as possible. However, only 59 patients had no missing data. Therefore, the number of patients with a full dataset of 
study using multicenter clinical data. Second, although the mean follow-up time was 33 months, the time to obtain the LastJOA was different. Increasing the number of enrolled patients in a future study will allow us to apply the BN model for each year of follow-up. The imaging status such as severity of the T2 signal change in the cervical cord on MRI was not considered in the present study. Adding this imaging variable analyzed by convolutional neural networks may help to improve the BN model's predictive ability.

The predictive model used in the present study may be controversial from the viewpoint of confidence. However, other statistical analyses or predictive models have not provided greater accuracy or better intuitive results than the final $B N$ with same number of patients and factors. Using information such as sex, dementia, and the PreJOA, the final BN may help to anticipate the probability of the LastJOA after posterior decompressive surgery for each patient with CSM.

\section{Conclusions}

The causal predictors of surgical outcome for CSM were sex, dementia, and preoperative JOA score. Use of the PGM with the Bayesian network may be useful personalized medicine tool for predicting the outcome for each patient with CSM.

Keywords: Bayesian network; cervical myelopathy; graphical model; prediction; probability

\section{Conclusion}

Sex, dementia, and PreJOA are causal factors for the LastJOA after posterior decompressive surgery for patients with CSM. Although additional information may be needed to improve both the learning of the $\mathrm{BN}$ structure and the rate of prediction, the BN structure may be useful for predicting the probabilities for clinical outcomes for each patient who undergoes posterior decompressive surgery.

\section{Abbreviations}

ASIA, American Spinal Injury Association; BANJO, Bayesian Network Inference with Java Objects ; BMI, body mass index ; $B N$, Bayesian networks; $C R$, cord compression ratio observed on the preoperative cervical MRI; CSM, cervical spondylotic myelopathy; DM, diabetes mellitus; JOA, Japanese Orthopaedic Association; LASSO, least absolute shrinkage and selection operator; LastJOA, last follow-up JOA score ; LOOCV, leave-one-out cross-validation; MB, Markov blanket; OPLL, ossification of the posterior longitudinal ligament; PGM, probabilistic graphical model; PreJOA, preoperative JOA score

\section{Declarations}

\section{Declarations}

Ethics approval and consent to participate: The consents from enrolled patients in the present study were not required because we reviewed the electrical medical records without contacts with patients, 
retrospectively. We received the approval from Boramae Hospital Institutional Review Board (number302020-20) with exemption from consent.

Consent for publication: Not applicable

Availability of data and materials: The datasets used and/or analyzed during the current study are available from the corresponding author on reasonable request.

Competing interests: The authors declare that they have no competing interests

Funding: No funding

Authors' contributions: SB Park analyzed and interpreted the patient data. Also, he was a major contributor in writing the manuscript. CW YOO interpreted the patient data and contributed the study design. DA shin and SH Lee were contributors in writing the manuscript. HJ Yang analyzed and interpreted the patient data. And all authors have read and approved the manuscript

Acknowledgements: Not applicable

\section{References}

1. Salem HM, Salem KM, Burget F, Bommireddy R, Klezl Z. Cervical spondylotic myelopathy: the prediction of outcome following surgical intervention in 93 patients using T1- and T2-weighted MRI scans. Eur Spine J. 2015;24(12):2930-35.

2. Bond M, Mclntosh G, Fisher C, Jacobs B, Johnson M, Bailey CS, et al. Treatment of Mild Cervical Myelopathy: Factors Associated With Decision for Surgical Intervention. Spine (Phila Pa 1976). 2019;44(22):1606-12.

3. Sharifi B, Mclntosh G, Fisher C, Jacobs WB, Johnson M, Bailey CS, et al. Consultation and Surgical Wait Times in Cervical Spondylotic Myelopathy. Can J Neurol Sci. 2019;46(4):430-5.

4. Karadimas SK, Erwin WM, Ely CG, Dettori JR, Fehlings MG. Pathophysiology and natural history of cervical spondylotic myelopathy. Spine (Phila Pa 1976). 2013;38(22 Suppl 1):21-36.

5. Sadasivan KK, Reddy RP, Albright JA. The natural history of cervical spondylotic myelopathy. Yale J Biol Med. 1993;66(3):235-42.

6. Fehlings MG, Arvin B. Surgical management of cervical degenerative disease: the evidence related to indications, impact, and outcome. J Neurosurg Spine. 2009;11(2):97-100.

7. Nagai T, Takahashi Y, Endo K, Ikegami R, Ueno R, Yamamoto K. Analysis of spastic gait in cervical myelopathy: Linking compression ratio to spatiotemporal and pedobarographic parameters. Gait Posture. 2018;59:152-6.

8. Son DK, Son DW, Song GS, Lee SW. Effectiveness of the laminoplasty in the elderly patients with cervical spondylotic myelopathy. Korean J Spine. 2014;11(2):39-44. 
9. Sun LQ, Li M, Li YM. Predictors for Surgical Outcome of Laminoplasty for Cervical Spondylotic Myelopathy. World Neurosurg. 2016;94:89-96.

10. Tetreault L, Palubiski LM, Kryshtalskyj M, Idler RK, Martin AR, Ganau M, et al. Significant Predictors of Outcome Following Surgery for the Treatment of Degenerative Cervical Myelopathy: A Systematic Review of the Literature. Neurosurg Clin N Am. 2018;29(1):115-27 e135.

11. Tetreault LA, Cote P, Kopjar B, Arnold P, Fehlings MG. A clinical prediction model to assess surgical outcome in patients with cervical spondylotic myelopathy: internal and external validations using the prospective multicenter AOSpine North American and international datasets of 743 patients. Spine J. 2015;15(3):388-97.

12. Shen $\mathrm{C}, \mathrm{Xu} \mathrm{H}, \mathrm{Xu} \mathrm{B}$, et al. Value of conventional MRI and diffusion tensor imaging parameters in predicting surgical outcome in patients with degenerative cervical myelopathy. $\mathrm{J}$ Back Musculoskelet Rehabil. 2018;31(3):525-32.

13. Takenaka S, Aono H. Prediction of Postoperative Clinical Recovery of Drop Foot Attributable to Lumbar Degenerative Diseases, via a Bayesian Network. Clin Orthop Relat Res. 2017;475(3):872-80.

14. Harrell FE Jr, Lee KL, Mark DB. Multivariable prognostic models: issues in developing models, evaluating assumptions and adequacy, and measuring and reducing errors. Stat Med. 1996;15(4):361-87.

15. Arora P, Boyne D, Slater JJ, Gupta A, Brenner DR, Druzdzel MJ. Bayesian Networks for Risk Prediction Using Real-World Data: A Tool for Precision Medicine. Value Health. 2019;22(4):439-45.

16. Onisko A, Druzdzel MJ, Austin RM. How to interpret the results of medical time series data analysis: Classical statistical approaches versus dynamic Bayesian network modeling. J Pathol Inform. 2016;7:50.

17. Yoo C, Ramirez L, Liuzzi J. Big data analysis using modern statistical and machine learning methods in medicine. Int Neurourol J. 2014;18(2):50-7.

18. Suzuki T, Shimoda T, Takahashi N, Tsutsumi K, Samukawa M, Yoshimura S, et al. Factors Affecting Bone Mineral Density Among Snowy Region Residents in Japan: Analysis Using Multiple Linear Regression and Bayesian Network Model. Interact J Med Res. 2018;7(1):e10.

19. Tetreault L, Wilson JR, Kotter MR, Nouri A, Côté P, Kopjar B, et al. Predicting the minimum clinically important difference in patients undergoing surgery for the treatment of degenerative cervical myelopathy. Neurosurg Focus. 2016;40(6):E14.

20. Park SB, Chung CK, Gonzalez E, Yoo C. Causal Inference Network of Genes Related with Bone Metastasis of Breast Cancer and Osteoblasts Using Causal Bayesian Networks. J Bone Metab. 2018;25(4):251-66.

21. Pumberger M, Schmidt H, Putzier M. Spinal Deformity Surgery: A Critical Review of Alignment and Balance. Asian Spine J. 2018;12(4):775-83.

22. Agostinho NB, Machado KS, Werhli AV. Inference of regulatory networks with a convergence improved MCMC sampler. BMC Bioinformatics. 2015;16:306. 
23. Khachatryan T, Robinson JS. The possible impact of cervical stenosis on cephalad neuronal dysfunction. Med Hypotheses. 2018;118:13-8.

24. Yang S, Nguyen ND, Center JR, Eisman JA, Nguyen TV. Association between hypertension and fragility fracture: a longitudinal study. Osteoporos Int. 2014;25(1):97-103.

\section{Figures}

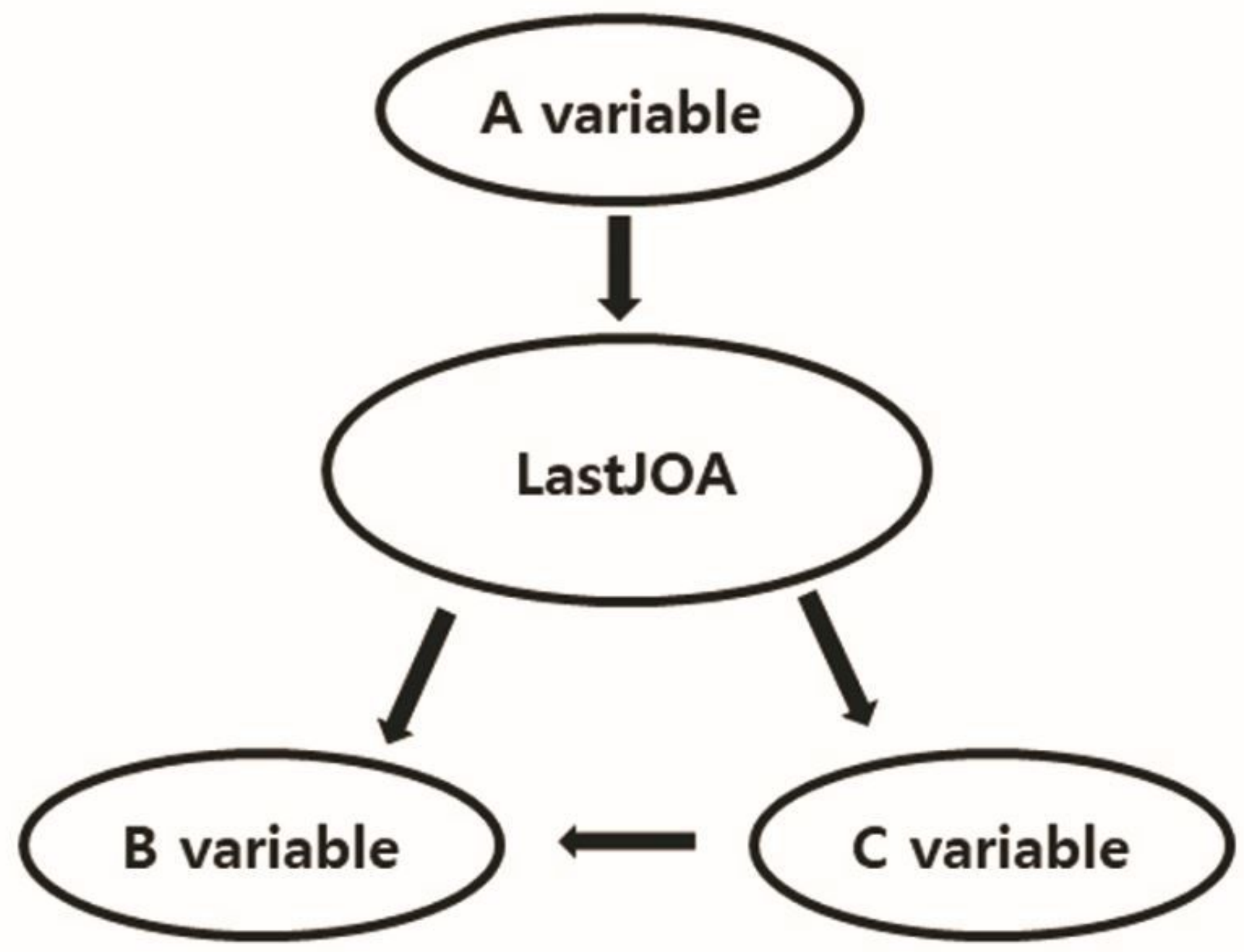

Figure 1

An example of Bayesian networks structure. Variable A influences the likelihood of the LastJOA, which influences on variables $B$ and $C$. The conditional independence between nodes shows as the probability that the expression of variables $B$ and $C$ is not influenced by variable $A$ given information for the LastJOA. LastJOA = last follow-up score of Japanese Orthopaedic Association 


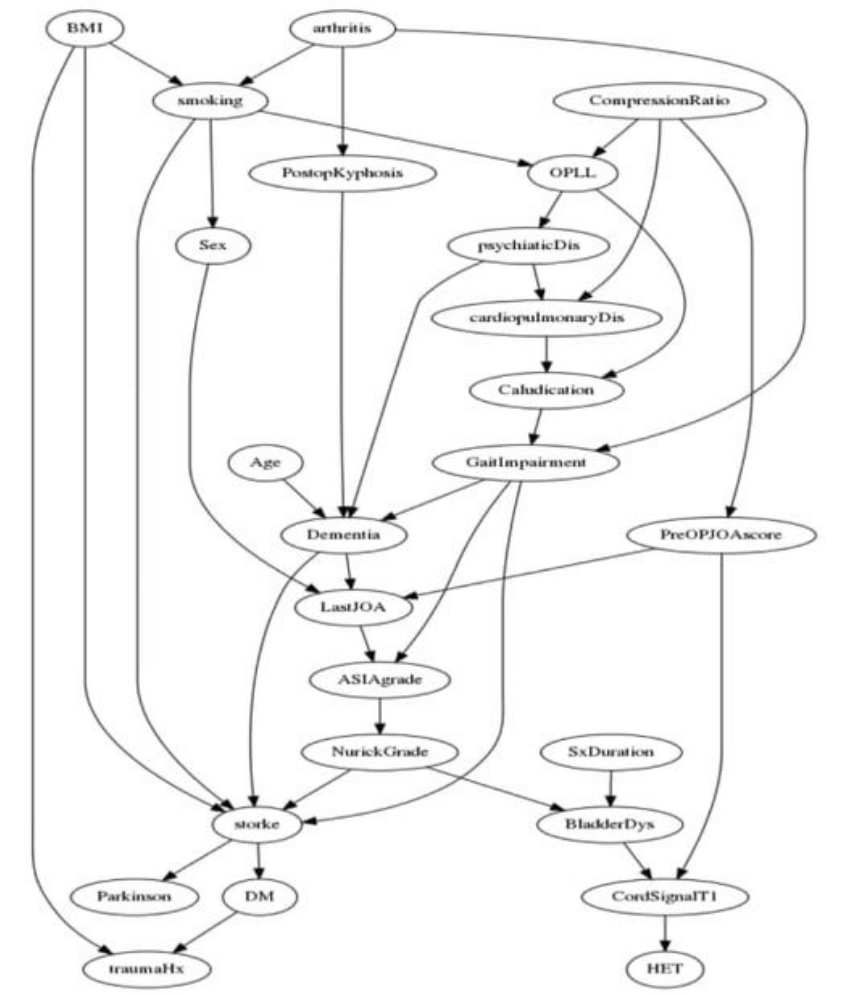

A

\section{Figure 2}

First and second Bayesian networks (BNs) relevant to the LastJOA Panel $A$ is shows the first BN with the 25 variables. Sex, dementia, and PreJOA are the parent variables of the LastJOA and the ASIA grade is only child variable of the LastJOA. Panel $B$ shows that the second $B N$ comprises 16 variables that are within the second-degree Markov Blanket of the LastJOA variable in the first BN. In the second BN, the parent variables of LastJOA are sex, dementia, PreJOA, and the child variable is ASIA grade. The BANJO score were -850.7081 and -564.9072 for the first and second $B N s$, respectively. ASIA $=$ American Spinal Injury Association; $\mathrm{BMI}$ = body mass index, $\mathrm{BN}=$ Bayesian network; $\mathrm{DM}=$ diabetes mellitus; LastJOA = last follow-up score of Japanese Orthopaedic Association; PreJOA = preoperative score of Japanese Orthopaedic Association; OPLL = ossification of posterior longitudinal ligament (OPLL) 


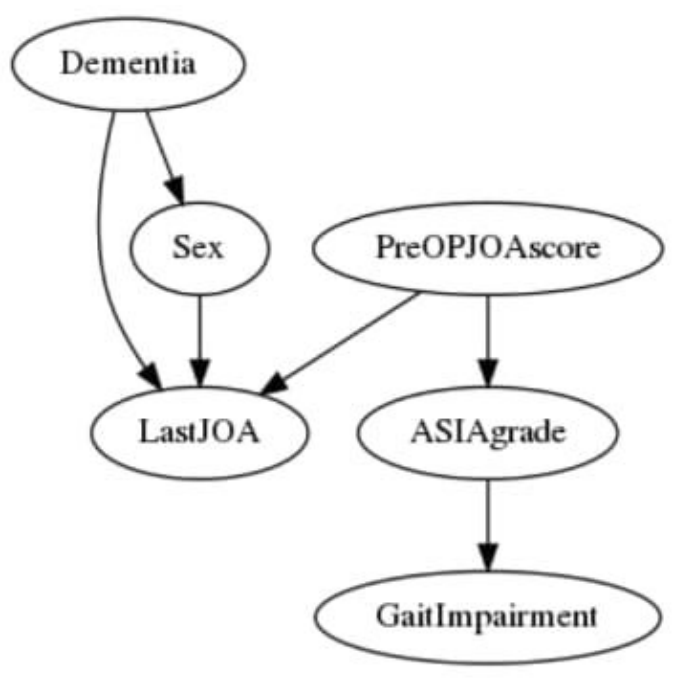

A

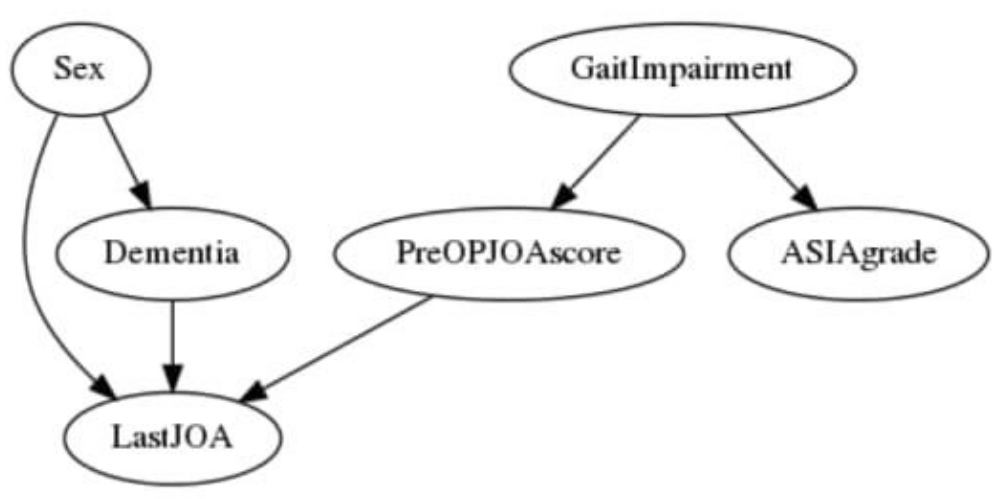

B

\section{Figure 3}

Bayesian network via Banjo analysis (A) and Order algorithm and structural code (B) with variables relevant to the LastJOA. The connection between variables and the direction of the arrows between them are similar in two structures. The log-likelihood score in the order algorithm and structure code was superior to that in the last BANJO analysis (-214.384 vs. -222.6719$)$. ASIA = American Spinal Injury Association; LastJOA = last follow-up score of Japanese Orthopaedic Association; PreJOA = preoperative score of Japanese Orthopaedic Association

A
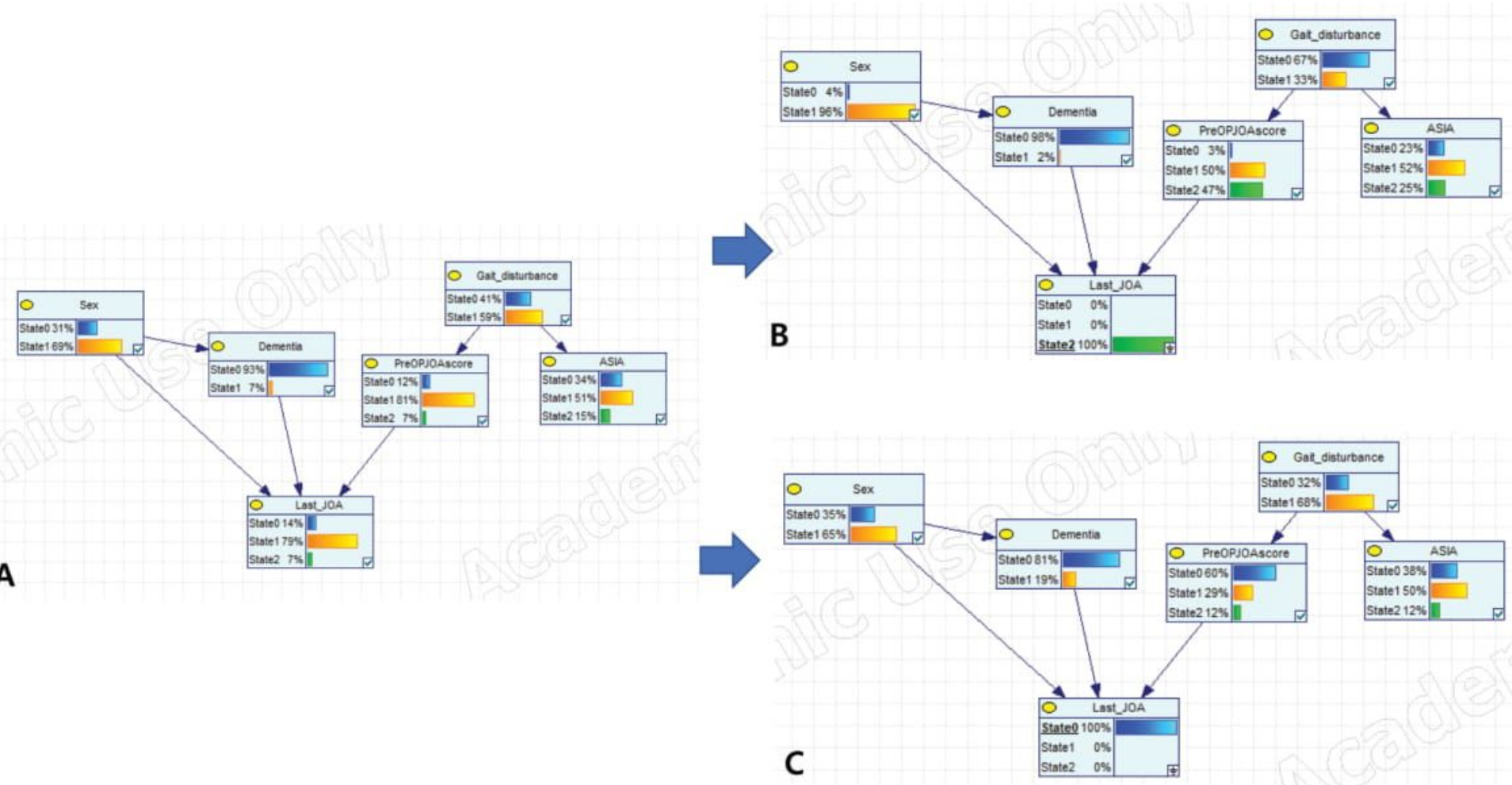

Figure 4 
Final Bayesian network structure and different conditions for the LastJOA obtained using GeNle. Panel A shows the Bayesian structure learned with the existing data set using GeNle. State 0 and 1 in the Gait_disturbance, Dementia, and Sex nodes represent the probability of the occurrence of the comorbidity for the first two variables or, male. States 0,1 , and 2 in other nodes represent the discretized values of 0 , 1 , and 2 , respectively. Panels $B$ and $C$ show that the change in the states in other variables after the LastJOA was conditioned as state 0 or 2 . ASIA = American Spinal Injury Association; LastJOA = last follow-up score of Japanese Orthopaedic Association; PreJOA = preoperative score of Japanese Orthopaedic Association
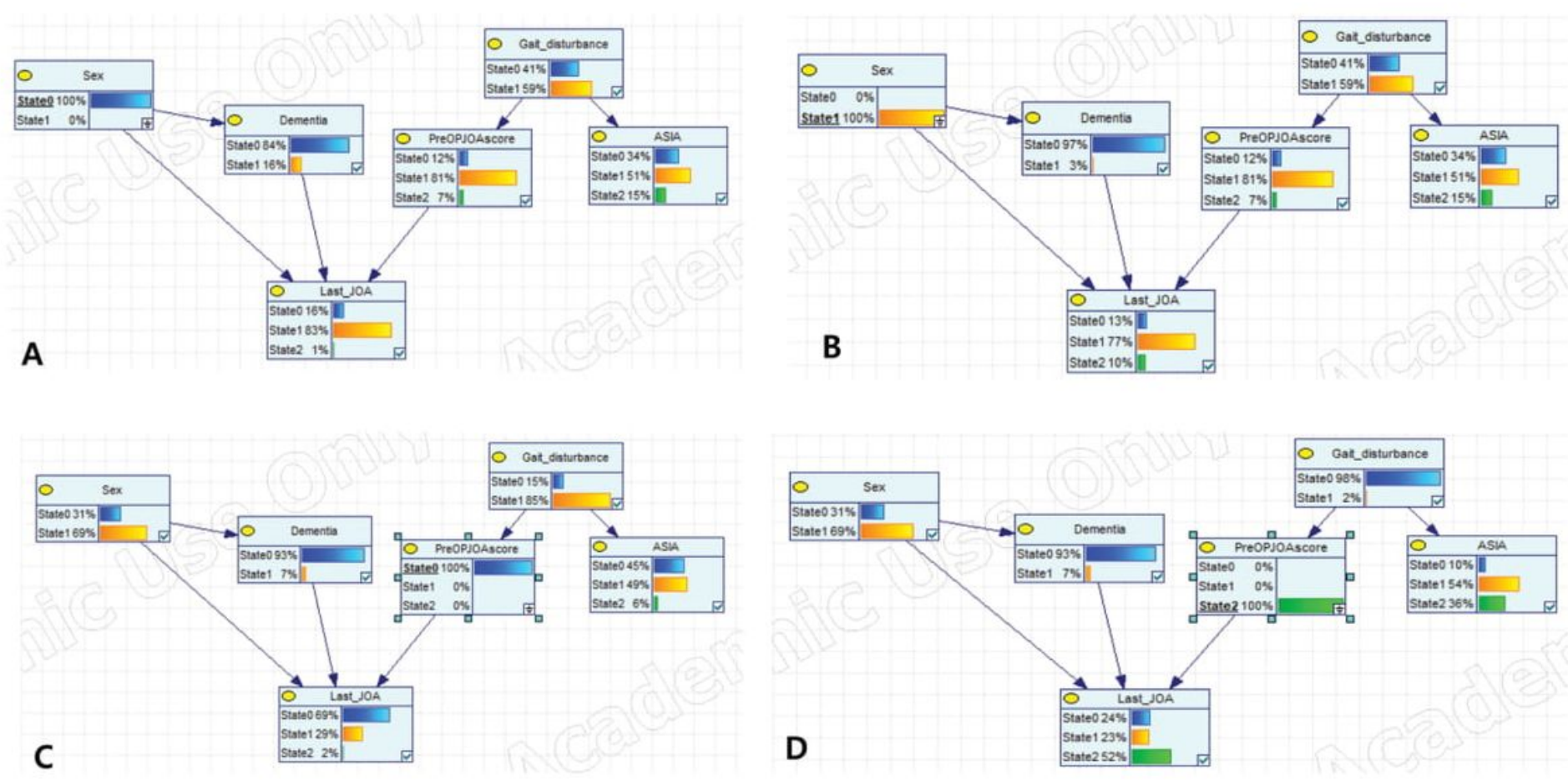

\section{Figure 5}

Bayesian network structures according to different conditions of Sex and the PreJOA. The figure shows the change in probability of the LastJOA in the different conditions according to the probabilities of Sex ( $A$ and $B$ ) and the PreJOA ( $C$ and $D)$. ASIA = American Spinal Injury Association; LastJOA = last follow-up score of Japanese Orthopaedic Association; PreJOA = preoperative score of Japanese Orthopaedic Association 

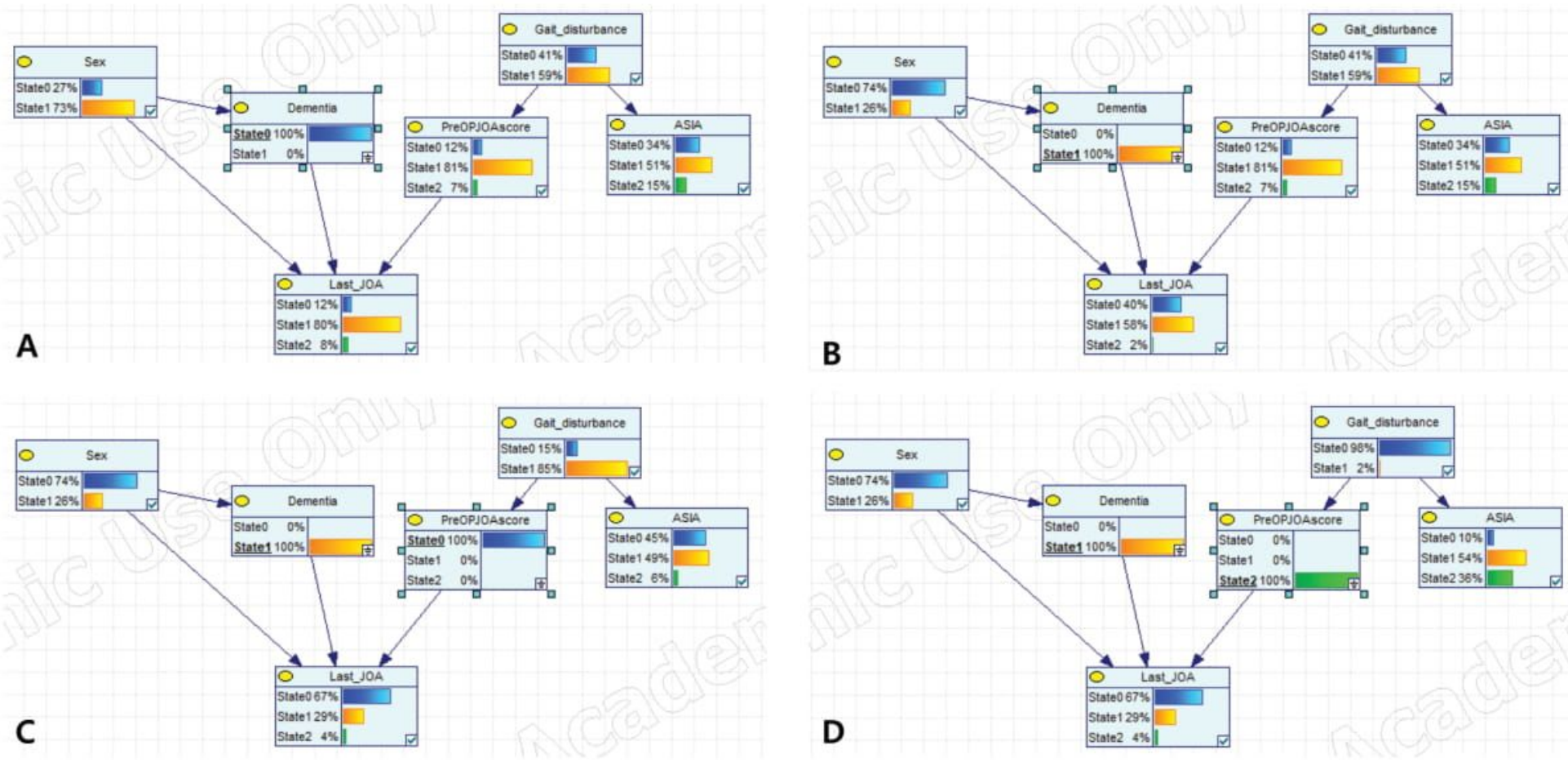

Figure 6

Bayesian network structures according to different conditions for the dementia node formation. Panels A and $B$ show the change in probability of the LastJOA in the different conditions for the probability of dementia. Panels $C$ and $D$ show the change in probability for the LastJOA for different conditions according to the probabilities of dementia and conditioned dementia, simultaneously. ASIA = American Spinal Injury Association; LastJOA = last follow-up score of Japanese Orthopaedic Association; PreJOA = preoperative score of Japanese Orthopaedic Association
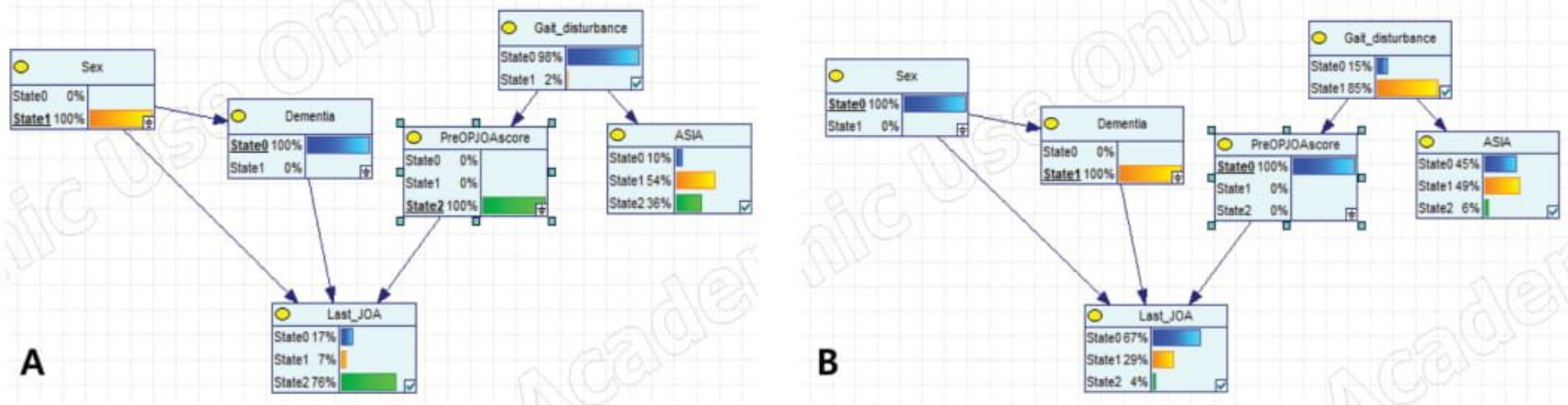

Figure 7

Bayesian network structures under different conditions of sex, dementia and the PreJOA, simultaneously. The figure shows the best $(A)$ and worst $(B)$ combinations of parent nodes for the LastJOA node in the Bayesian network structures. ASIA = American Spinal Injury Association; LastJOA = last follow-up score of Japanese Orthopaedic Association; PreJOA = preoperative score of Japanese Orthopaedic Association 